\title{
Pathologic features and expression of heat shock protein 47 in the nasal mucosa and lacrimal sac: does it influence the surgical outcome of endoscopic endonasal dacryocystorhinostomy?
}

\author{
Jinhwan Park ${ }^{1} \cdot$ Joonsik Lee $^{2} \cdot$ Sehyun Baek ${ }^{1}$
}

Received: 18 December 2017 / Revised: 7 April 2018 / Accepted: 10 April 2018 / Published online: 18 May 2018

(c) The Royal College of Ophthalmologists 2018

\begin{abstract}
Purpose To correlate the pathologic features and heat shock protein 47 (HSP47) expression in the nasal mucosa and lacrimal sac with the surgical outcomes of endoscopic endonasal dacryocystorhinostomy (EDCR).

Methods Specimens of the nasal mucosa and lacrimal sac over the rhinostomy site were collected during the operation. Haematoxylin-eosin and immunohistochemical staining were performed to determine the pathologic features (inflammation, fibrosis, squamous metaplasia) and the expression of HSP47 in the epithelium and sub-epithelial glands of the nasal mucosa and lacrimal sac. The success or failure of EDCR was determined 6 months after surgery.

Results A total of 30 patients (30 eyes) were included in this study. Inflammation and squamous metaplasia of the nasal mucosa were not associated with the surgical outcome of EDCR ( $p=0.485$ and 0.069 , respectively), but there was an association with fibrosis of the nasal mucosa $(p=0.003)$. In addition, HSP47 in the nasal mucosa was associated with the surgical outcomes $(p=0.005)$. Inflammation of the lacrimal sac was not associated with the surgical outcome of EDCR $(p=$ 0.509 ), but fibrosis and squamous metaplasia of the lacrimal sac were $(p=0.005$ and 0.008 , respectively). Additionally, HSP47 in the lacrimal sac was associated with surgical outcomes $(p<0.001)$.

Conclusion Fibrosis and squamous metaplasia of the nasal mucosa and lacrimal sac lowered the success rate of EDCR. HSP47 also lowered the surgical success rate. Fibrosis was correlated with the expression of HSP47.
\end{abstract}

\section{Introduction}

Nasolacrimal duct obstruction (NLDO) is a disorder with epiphora as a main symptom, and dacryocystorhinostomy (DCR) is a standard treatment for NLDO [1]. DCR can be performed by the existing classical method via the skin (transcutaneous) or via the nasal cavity (transnasal) using an endoscope [2]. There are various methods for the formation of an ostium in endoscopic endonasal dacryocystorhinostomy (EDCR), and the success rate exceeds $80 \%$ [3]. In EDCR, the rates of adhesion of ostium and the formation of scars and granulomas range from 0.6 to $11 \%$ [4], and

Sehyun Baek

shbaek6534@korea.ac.kr

1 Department of Ophthalmology, Korea University College of Medicine, Seoul, Korea

2 Yesan Seoul Eye Clinic, Yesan-gun, Chungcheongnam-do, Korea reducing these complications is important for increasing the success rate of the surgery. In addition, if NLDO continues as the inflammation of the lacrimal sac and nasolacrimal duct (NLD) becomes chronic, fibrosis and squamous metaplasia occur, and this histological change aggravates NLDO [5].

Several recent studies have shown that heat shock protein 47 (HSP47) is closely associated with the fibrosis of a tissue [6]. HSP47 is a collagen-specific protein, and it has a crucial role in the fibrosis of collagen [6]. In addition, DCR outcomes can be predicted based on HSP47 expression and the degree of squamous metaplasia in the nasal mucosa [7].

The present study investigates inflammation, fibrosis, the degree of squamous metaplasia, and the degree of HSP47 expression in nasal mucosa and lacrimal sac tissue in patients who underwent EDCR for NLDO, and examines the impact of these factors on surgical outcomes. 


\section{Patients and methods}

\section{Patients}

This study was conducted prospectively in 30 patients who were diagnosed with primary acquired nasolacrimal duct obstruction (PANDO) and underwent endoscopic endonasal DCR. Patients with functional NLDO or secondary NLDO due to an anatomical anomaly were excluded. This study obtained approval from the Korea University Guro Hospital Institutional Review Board and observed the conditions of the Helsinki Declaration.

\section{Surgical method}

EDCR was performed using a 4.0-mm 00 nasal endoscope under general anesthesia with endotracheal intubation by one oculoplastic surgeon (SHB). The nose (middle meatus) was packed with pledgets soaked in a $4 \%$ lidocaine and 1:1000 epinephrine solution for 30-60 min. First, a $5 \times 5 \mathrm{~mm}$ section of nasal mucosa around the anterior side of the uncinate process and the operculum of the middle turbinate, which is the anatomical indicator of the lacrimal sac, was removed using a sickle knife for immunopathologic examination. After this, the operation site in the nasal mucosa was injected with a $1: 100,000$ epinephrine solution from the projection of the lacrimal bone to the unciform apophysis. The nasal mucosa around the $5 \times 5 \mathrm{~mm}$ excision was further removed using a monopolar, and the line of demarcation between the lacrimal bone and the maxillary bone surrounding the lacrimal sac was exposed completely. Then, using Kerrison Rongeurs, the line of demarcation between the lacrimal bone and the maxillary bone was removed to form a bony ostium, and the lacrimal sac was completely exposed. Using a sickle knife, an incision of the lacrimal sac was made, and the medial wall of the lacrimal sac was removed using an ethmoid forceps. Half of the collected nasal mucosa and lacrimal sac were stored in frozen nitrogen, and the other half were stored in a $4 \%$ formalin solution. Using the collected nasal mucosa and lacrimal sac, HSP47 immunochemical stains and Hematoxylin \& Eosin stains (H\&E stain) were carried out. After bicanaliculus intubation (Bika; FCI Ophthalmics, Pembroke, MA, USA) through the upper and lower canaliculi, synthetic polyurethane foam (Nasopore; Polyganics, Rozenburglaan, Groningen, Netherlands) or expandable polyvinyl acetate (Merocel; Medtronic Xomed, Jacksonville, FL, USA) $(1 \mathrm{~cm} \times 1 \mathrm{~cm} \times 1 \mathrm{~cm})$ was packed at the anastomosis site. Nasopore and Merocel were then soaked in a gentamycin solution.

\section{HSP47 immunochemical staining}

Tissues kept in a freezer were fixed for $12 \mathrm{~h}$ in $4 \%$ paraformaldehyde solution and embedded in paraffin. The embedded tissues were sliced at $5 \mu \mathrm{m}$ thickness, and then the paraffin was removed with xylene. Sections were treated in ethanol by stages and dissolved in distilled water. After reaction with a protein blocking agent for $15 \mathrm{~min}$ to inhibit endogenous peroxidase activity, they were treated with $3 \%$ hydrogen peroxide solution for $10 \mathrm{~min}$ and washed with phosphate-buffered saline (PBS) for $5 \mathrm{~min}$. They were then reacted in primary antibody (ab77609, Abcam, Cambridge, UK) to HSP47 diluted at $1: 250$ at $4{ }^{\circ} \mathrm{C}$ overnight. After washing sections with $\mathrm{PBS}$, they were reacted with a horseradish peroxidase kit (DAKO LSAB 2 System, HRP Kit Cat No. K0675, Carpinteria, CA, USA) for $30 \mathrm{~min}$, washed with PBS, and reacted with DAB-chromogen (Zymed Cat No.00-2020 DAB substrate kit, Carlsbad, CA, USA) for color development and contrast dye in $0.5 \%$ methyl green for $3 \mathrm{~min}$. Sections were imaged under an optical microscope.

\section{H\&E stain}

Paraffin blocks of each tissue were made with $5 \mu \mathrm{m}$ sections, fixed at $60{ }^{\circ} \mathrm{C}$ for one hour on a slide, and deparaffinized using xylene. The deparaffinized slide was placed into a beaker in order to be submerged in $0.01 \mathrm{M}$ citrate solution, heated for $10 \mathrm{~min}$ in a microwave oven, cooled sufficiently, and cleaned with distilled water and then with $3 \% \mathrm{H}_{2} \mathrm{O}_{2}$. After nuclear staining using hematoxylin, the slide was sealed to observe the tissues under a microscope.

\section{Histopathologic measurements}

Tissue samples were assessed for inflammation, fibrosis, and squamous metaplasia. For the degree of inflammation, no inflammation was classified as normal; inflammation limited to $1 / 3$ rd of the upper part of the sub-epithelial layer thickness of lacrimal sac and nasal mucosa was classified as mild; invasion up to $2 / 3 \mathrm{rd}$ was classified as moderate; and invasion of the full thickness was classified as severe. No fibrosis was classified as normal; fibrosis limited to $1 / 3$ rd of the upper part of the sub-epithelial layer was classified as mild; invasion up to $2 / 3 \mathrm{rd}$ was classified as moderate; and invasion of full thickness was classified as severe [5, 8]. For squamous metaplasia, no squamous metaplasia was classified as normal; squamous metaplasia limited to $1 / 4$ th of the sub-epithelial layer was classified as mild; invasion up to 1/ 2 was classified as moderate; and invasion over $1 / 2$ was classified as severe. When evaluating HSP47 expression, the amount of staining of epithelial cells and interstitial cells 
Table 1 Histological features, HSP47, and surgical outcomes in the nasal mucosa and lacrimal sac

\begin{tabular}{|c|c|c|c|c|c|c|}
\hline & \multicolumn{3}{|c|}{ Nasal mucosa } & \multicolumn{3}{|l|}{ Lacrimal sac } \\
\hline & Success & Failure & $p^{*}$ & Success & Failure & $p^{*}$ \\
\hline & \multicolumn{3}{|c|}{ Patient number $(\%)$} & \multicolumn{3}{|c|}{ Patient number $(\%)$} \\
\hline \multicolumn{7}{|l|}{ Inflammation } \\
\hline No & $3(100 \%)$ & $0(0 \%)$ & 0.485 & $5(62.5 \%)$ & $3(37.5 \%)$ & 0.509 \\
\hline Mild & $7(77.7 \%)$ & $2(22.3 \%)$ & & $10(100 \%)$ & $0(0 \%)$ & \\
\hline Moderate & $8(72.7 \%)$ & $3(27.3 \%)$ & & $3(100 \%)$ & $0(0 \%)$ & \\
\hline Severe & $6(85.7 \%)$ & $1(14.3 \%)$ & & $6(66.6 \%)$ & $3(33.4 \%)$ & \\
\hline \multicolumn{7}{|l|}{ Fibrosis } \\
\hline No & $15(100 \%)$ & $0(0 \%)$ & 0.003 & $14(93.3 \%)$ & $1(6.7 \%)$ & 0.005 \\
\hline Mild & $9(64.2 \%)$ & $5(35.8 \%)$ & & $10(83.3 \%)$ & $2(16.7 \%)$ & \\
\hline Moderate & $0(0 \%)$ & $1(100 \%)$ & & $0(0 \%)$ & $3(100 \%)$ & \\
\hline Severe & $0(0 \%)$ & $0(100 \%)$ & & $0(0 \%)$ & $0(100 \%)$ & \\
\hline \multicolumn{7}{|c|}{ Sq. metaplasia } \\
\hline No & $18(90 \%)$ & $2(10 \%)$ & 0.069 & $16(100 \%)$ & $0(0 \%)$ & 0.008 \\
\hline Mild & $5(62.5 \%)$ & $3(37.5 \%)$ & & $6(60 \%)$ & $4(40 \%)$ & \\
\hline Moderate & $1(50 \%)$ & $1(50 \%)$ & & $2(50 \%)$ & $2(50 \%)$ & \\
\hline Severe & $0(0 \%)$ & $0(100 \%)$ & & $0(0 \%)$ & $0(100 \%)$ & \\
\hline \multicolumn{7}{|l|}{ Hsp47 exp } \\
\hline No & $9(100 \%)$ & $0(0 \%)$ & 0.005 & $15(100 \%)$ & $0(0 \%)$ & $<0.001$ \\
\hline Mild & $10(90.9 \%)$ & $1(9.1 \%)$ & & $9(90 \%)$ & $1(10 \%)$ & \\
\hline Moderate & $5(50 \%)$ & $5(50 \%)$ & & $0(0 \%)$ & $3(100 \%)$ & \\
\hline Severe & $0(0 \%)$ & $0(100 \%)$ & & $0(0 \%)$ & $2(100 \%)$ & \\
\hline
\end{tabular}

Sq. metaplasia squamous metaplasia

*Linear by linear association. $p$-values $<0.05$ were considered statistically significant was assessed, and no staining was classified as normal; staining less than $25 \%$ was classified as mild; staining more than $25 \%$ and $<50 \%$ was classified as moderate; and staining more than $50 \%$ was classified as severe [7].

\section{Surgical outcomes}

Follow-up observations after DCR were conducted 1 week, 1 month, 3 months, and 6 months after surgery, and nasal endoscopy was conducted at each follow-up. During the final follow-up, which was conducted 6 months later, epiphora was assessed, and a Munk scale score lower than 1 point (little wiping of tears) was defined as success, while higher than 2 points (wiping tears two to four times a day) was classified as failure [9]. Histological characteristics of the nasal mucosa and lacrimal sac (inflammation, fibrosis, and squamous metaplasia) were compared with regard to HSP47 expression and surgical outcomes, and correlations were investigated. In addition, in order to investigate the correlation between granuloma size and HSP47 expression, granulomas smaller than $1 / 3$ rd of the ostium region were classified as mild; those more than $1 / 3$ rd and less than $1 / 2$ the ostium size were classified as moderate; and those that encompassed more than $1 / 2$ the ostium size were classified as severe.

\section{Statistical analysis}

The data were analyzed using the Statistical Package for the Social Sciences version 21.0 (SPSS Inc, Chicago, IL, USA). Linear by linear association $(2 \times \mathrm{k}$ table) was used to analyze the relationship between two variables. $p$-values $<0.05$ were considered statistically significant.

\section{Results}

Of the 30 patients (30 eyes), 14 were men and 16 were women. The average age of the men was $56.8 \pm 10.1$ years, and that of the women was $55.0 \pm 11.8$ years. Six months after the surgery, 24 patients were classified as surgical successes $(80 \%)$, and $6(20 \%)$ were classified as surgical failures.

\section{Finding of H\&E staining in the nasal mucosa}

Three of 30 patients did not show any inflammation; 9 had mild inflammation; 11 had moderate inflammation; and 7 had severe inflammation (Table 1) (Fig. 1). All 3 patients who did not show any inflammation (100\%) showed surgical success. Seven $(77.7 \%)$ of nine with mild 
Fig. $1 \mathrm{H} \& \mathrm{E}$ staining of the nasal mucosa and lacrimal sac. a

Normal pseudostratified columnar epithelium of nasal mucosa $(\times 400)$. b Mild inflammation of the nasal mucosa (loose lymphocyte and neutrophil aggregation is shown) (×200). c Moderate inflammation of the lacrimal sac (inflammatory cells are concentrated in the superior $1 / 2$ of the submucosa) $(\times 200)$. $\mathbf{d}$ Mild squamous metaplasia of the lacrimal sac (stratified squamous epithelium is shown) $(\times 200)$. Immunohistochemical staning for heat shock protein 47 ( $\times 400)$. e Mild staining of the nasal mucosa. f Moderate staining of the lacrimal sac
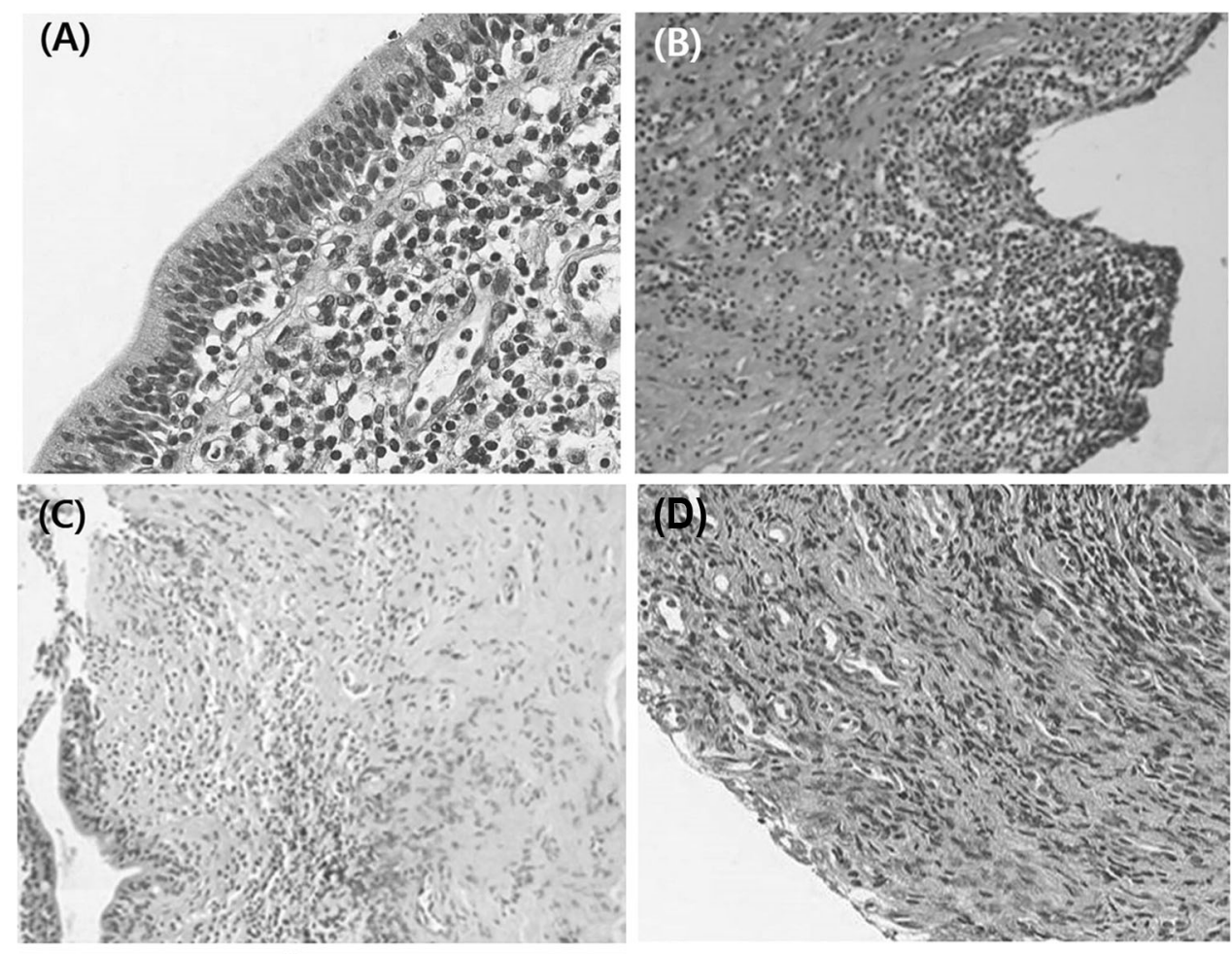

(E)
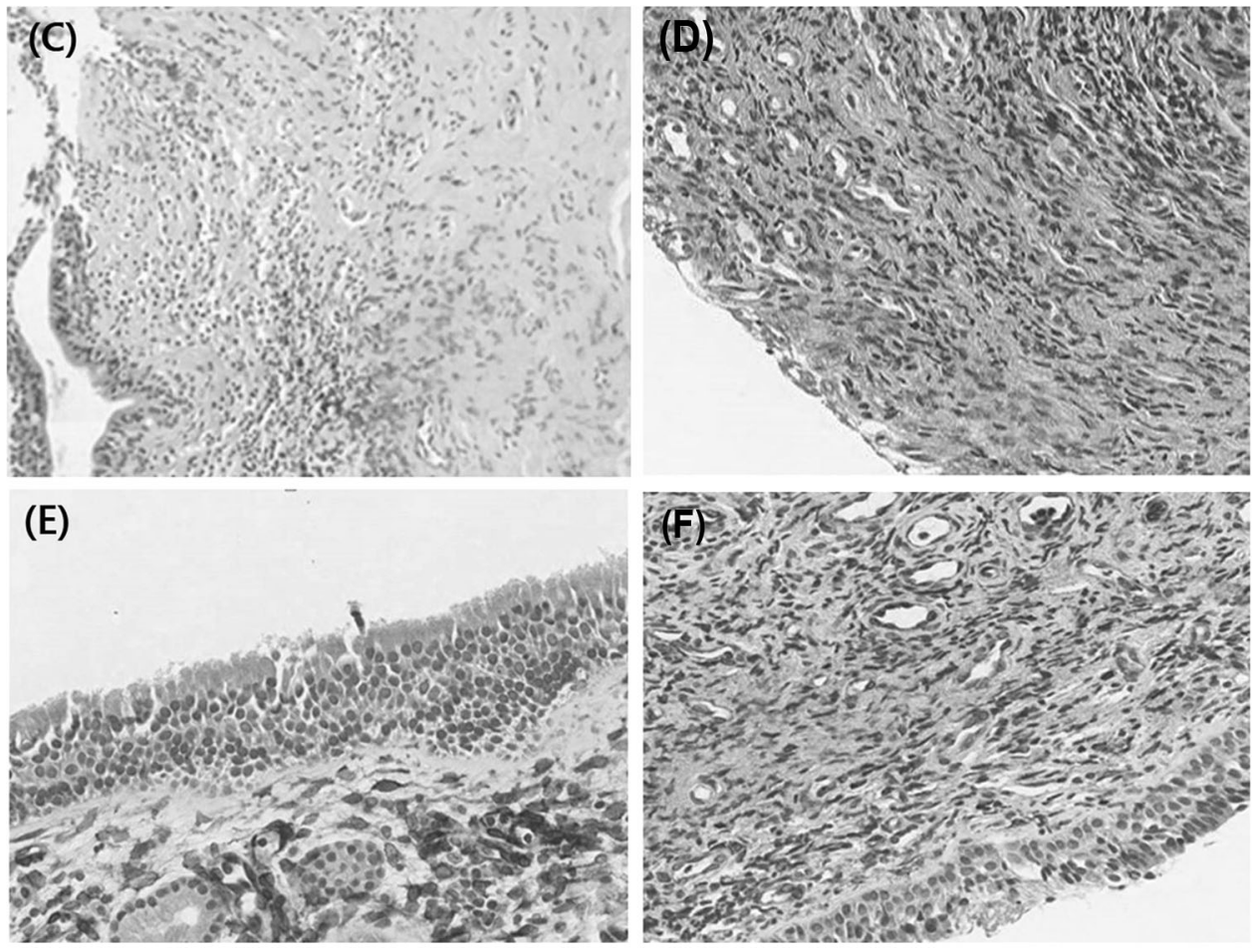

inflammation, eight $(72.7 \%)$ of eleven with moderate inflammation, and six (85.7\%) of seven patients with severe inflammation displayed surgical success. There was no significant correlation between the degree of nasal mucosa inflammation and the success rate of surgery $(p=$ $0.485)$.

In totally, 15 of the 30 patients did not show fibrosis, with 14 showing mild fibrosis and 1 showing moderate fibrosis (Table 1). All 15 patients (100\%) who did not have fibrosis showed surgical success, while $9(64.2 \%)$ of the 14 who showed mild fibrosis and the 1 who showed moderate fibrosis showed surgical failure. A high degree of fibrosis was significantly correlated with a decline in the success rate of the surgery $(p=0.003)$.

Squamous metaplasia appeared in 10 of 30 patients, and 8 patients showed mild squamous metaplasia, while 2 showed moderate squamous metaplasia (Table 1). Surgical success was seen in 18 (90\%) of 20 patients who did not have squamous metaplasia, 5 of 8 patients who showed mild squamous metaplasia (62.5\%), and $1(50 \%)$ of 2 patients who showed moderate squamous metaplasia. Higher degrees of squamous metaplasia were correlated with lower surgical success rates, but this was not statistically significant $(p=0.069)$.

\section{HSP47 expression in nasal mucosa}

HSP47 was expressed in 21 of 30 patients: mild expression was seen in 11 patients, moderate expression was seen in 10 , and there were no patients with severe expression (Table 1) (Fig. 1). All 9 patients (100\%) without HSP47 expression had surgical success, and $10(90.9 \%)$ of 11 who showed mild expression and $5(50 \%)$ of 10 who showed moderate expression had surgical success. Higher HSP47 expression was significantly correlated with lower surgical success rates $(p=0.005)$. 
Table 2 HSP47 expression and fibrosis in the nasal mucosa

\begin{tabular}{llllll}
\hline & \multicolumn{2}{l}{ Fibrosis (patient number) } & \multirow{2}{*}{$p^{*}$} \\
\cline { 2 - 5 } HSP47 expression (patient number) & No (15) & Mild (14) & Moderate (1) & Severe (0) & \\
\hline No (9) & 9 & 0 & 0 & 0 & $<0.001$ \\
Mild (11) & 6 & 5 & 0 & 0 & \\
Moderate (10) & 0 & 9 & 1 & 0 & \\
Severe (0) & 0 & 0 & 0 & 0 & \\
\hline
\end{tabular}

*Linear by linear association. $p$-values $<0.05$ were considered statistically significant

\begin{tabular}{llllll}
\hline & \multicolumn{2}{l}{ Fibrosis (patient number) } & \multirow{2}{*}{$p^{*}$} \\
\cline { 2 - 5 } HSP47 expression (patient number) & No (15) & Mild (12) & Moderate (3) & Severe (0) & \\
\hline No (15) & 14 & 1 & 0 & 0 & $<0.001$ \\
Mild (10) & 0 & 10 & 0 & 0 & \\
Moderate (3) & 1 & 1 & 1 & 0 & \\
Severe (2) & 0 & 0 & 2 & 0 & \\
\hline
\end{tabular}

* Linear by linear association. $p$-values $<0.05$ were considered statistically significant
Table 3 HSP47 expression and fibrosis in the lacrimal sac

\section{HSP47 expression in the lacrimal sac}

\section{Finding of H\&S stain in lacrimal sac}

Eight of 30 patients showed no inflammation, 10 showed mild inflammation, 3 showed moderate inflammation, and 9 showed severe inflammation (Table 1) (Fig. 1). Five $(62.5 \%)$ of eight patients without inflammation had surgical success, while ten patients with mild inflammation, three patients with moderate inflammation $(100 \%)$, and six of nine with severe inflammation $(66.6 \%)$ had surgical success. There was no significant correlation between the degree of lacrimal sac inflammation and the surgical success rate $(p=0.509)$.

From the total of 30 patients, 15 did not show fibrosis, 12 showed mild fibrosis, and the remaining 3 patients showed moderate fibrosis (Table 1). Of the patients who did not show fibrosis, 14 (93.3\%) had surgical success, while 10 (83.3\%) of 12 patients who showed mild fibrosis, and none $(0 \%)$ of three who showed moderate fibrosis had surgical success. Patients with a more severe degree of fibrosis tended to have significantly lower surgical success rates ( $p$ $=0.005$ ).

Sixteen of 30 patients did not show squamous metaplasia, 10 showed mild squamous metaplasia, and 4 showed moderate squamous metaplasia (Table 1) (Fig. 1). All 16 patients (100\%) who did not show squamous metaplasia had surgical success, while $6(60 \%)$ of 10 patients who showed mild squamous metaplasia and 2 $(50 \%)$ of 4 patients who showed moderate squamous metaplasia had surgical success. More severe squamous metaplasia was significantly correlated with surgical failure $(p=0.008)$.
Of 30 patients, mild expression was found in 10, moderate expression was found in 3 , severe expression was found in 2, and 15 patients did not show HSP47 expression (Table 1) (Fig. 1). In all 15 patients (100\%) who did not show HSP47 expression and in 9 (90\%) of 10 patients who showed mild expression, the surgery was successful. In contrast, the outcome of the surgery was failure in all three patients with moderate expression and in both patients with severe expression. Higher HSP47 expression was significantly correlated with lower surgical success rate $(p<0.001)$.

\section{Correlation between HSP47 expression and fibrosis}

All nine patients who did not show HSP47 expression in the nasal mucosa did not show fibrosis; 6 of 11 patients who showed mild HSP47 expression did not show fibrosis, and 5 showed mild fibrosis. Nine of 10 patients who showed moderate HSP47 expression showed mild fibrosis and 1 showed moderate fibrosis, and HSP47 expression in the nasal mucosa was significantly correlated with the degree of fibrosis in the nasal mucosa (Table 2) $(p<0.001)$.

Fibrosis was found in 14 of 15 patients who did not show HSP47 expression in the lacrimal sac, and the other 1 showed mild fibrosis. All 10 patients who showed mild HSP47 expression showed mild fibrosis. One of three patients who showed moderate HSP47 expression did not show fibrosis, while one showed mild fibrosis and one showed moderate fibrosis. There was a significant correlation between HSP47 expression in the lacrimal sac and 
Table 4 HSP47 expression in the nasal or lacrimal sac mucosa, and granuloma size over the osteotomy site in failed endo-DCR

\begin{tabular}{|c|c|c|c|c|}
\hline & \multicolumn{3}{|c|}{ Granuloma size (patient number) } & \multirow[t]{2}{*}{$p^{*}$} \\
\hline & Mild (3) & Moderate (3) & Severe $(0)$ & \\
\hline \multicolumn{5}{|c|}{$\begin{array}{l}\text { HSP } 47 \text { expression in } \\
\text { nasal mucosa (patient } \\
\text { number) }\end{array}$} \\
\hline No (9) & 0 & 0 & 0 & 0.17 \\
\hline Mild (11) & 0 & 1 & 0 & \\
\hline Moderate (10) & 3 & 2 & 0 & \\
\hline Severe $(0)$ & 0 & 0 & 0 & \\
\hline \multicolumn{5}{|c|}{$\begin{array}{l}\text { HSP47 expression in } \\
\text { lacrimal sac mucosa }\end{array}$} \\
\hline No (15) & 0 & 0 & 0 & 0.08 \\
\hline Mild (10) & 0 & 1 & 0 & \\
\hline Moderate (3) & 3 & 0 & 0 & \\
\hline Severe (2) & 0 & 2 & 0 & \\
\hline
\end{tabular}

*Linear by linear association. $p$-values $<0.05$ were considered statistically significant

the degree of fibrosis in the lacrimal sac (Table 3) $(p<$ 0.001 )

\section{Correlation between HSP47 expression and the granuloma size in the ostium region}

There was no statistically significant correlation between HSP47 expression in the nasal mucosa and lacrimal sac and the size of the granuloma formed in the ostium region (Table 4) ( $p=0.17$ and $p=0.08$, respectively).

\section{Discussion}

Surgical success of EDCR depends on the opening of the region where an ostium is formed, and post-surgical granuloma, fibrosis, and adhesion formed in the ostium region are the most common causes for surgical failure [3]. Techniques and methods to reduce these post-surgical failures have been introduced, and typical methods include a mitomycin $\mathrm{C}$ drop in the nasal and lacrimal sac mucosa in the region where an ostium is formed [10] and packing using absorbable filling in the region where an ostium is formed [11].

There are several previous studies that investigated pathological observations during the process of DCR in patients with PANDO. Ciftci et al. [12] collected the lacrimal sac tissues of patients who underwent revision surgery after primary DCR failed and suggested chronic inflammation and excessive fibrosis on biopsy as the causes for ostium obstruction. Mauriello et al. [8] collected the nasal mucosa and lacrimal sacs of 44 NLDO patients in the surgical process and found chronic inflammation, excessive fibrosis, and goblet cell expression in the nasal mucosa and lacrimal sac. By confirming that these were not different from the observations of the inflammation and fibrosis of NLD epithelial cells, they reported that all inflammation of nasal mucosa, the lacrimal sac, and NLD epithelial mucosal tissue can contribute to NLDO. The above studies suggest that inflammation in the nasal mucosa, lacrimal sac, and NLD, as well as histological changes due to fibrosis, might act as partial causes for PANDO and ostium obstruction after DCR.

Recently, it has been found that HSP47 is closely associated with fibrosis $[13,14]$ and there is excessive HSP47 expression in keloid regions compared to the normal skin [13]. HSP47 is a collagen-specific reaction protein in the endoplasmic reticulum, which reacts to collagen precursor and disturbs the degeneration and cohesion of proteins in the processes of cohesion, formation, and transmission. It is a glycoprotein with a molecular weight of $47 \mathrm{kD}$ that is known to be helpful in bending procollagen, acts as a collagen precursor, and synthesizes different kinds of collagen. Most HSPs induce different types of target protein; however, collagen is solely formed by HSP47, which has been confirmed through many studies with fibrosis model animals and clinical tests $[15,16]$. Through an animal test, it was found that an antisense oligonucleotide that reduces the expression of HSP47 specifically from collagen can inhibit the accumulation of collagen in glomerulosclerosis and reduce scar reaction and fibrosis [17]. Smirnov et al. [7] investigated squamous metaplasia in the nasal mucosa and HSP47 expression collected from 30 PANDO patients in the process of EDCR and found that the surgical failure rate was higher in the group with squamous metaplasia than in the group without (failure rate: $3 \%$ and 14\%, respectively). Also, the degree of quantitative HSP47 expression was higher in the group with squamous metaplasia than in the group without, and squamous metaplasia affected the formation of granuloma in the ostium region after surgery. In addition, they reported that the degree of squamous metaplasia in the nasal mucosa in the region where an ostium would be formed before the surgery may affect the outcome by controlling HSP47 expression.

This study collected lacrimal sacs as well as the nasal mucosa of 30 PANDO patients in the region where an ostium was formed and analyzed HSP47 expression, squamous metaplasia, inflammation, and the degree of fibrosis in these two regions. First, the degree of inflammation did not affect the outcome of the surgery in squamous mucosa or the lacrimal sac $(p=0.485$ and $p=0.509$, respectively). This indicates that an inflammatory reaction does not have an adverse impact on the outcome of surgery until it causes fibrosis and squamous metaplasia, and it suggests that it is 
advantageous not to delay surgery after a PANDO diagnosis. No statistical significance was found in the nasal mucosa, but higher degrees of squamous metaplasia in the nasal mucosa and lacrimal sac were correlated with lower surgical success rates $(p=0.069$ and $p=0.008$, respectively). Eighteen of 20 patients without squamous metaplasia in the nasal mucosa had surgical success (90\%), while all 16 persons without squamous metaplasia in the lacrimal sac showed a surgical success (100\%). Fibrosis was significantly correlated with surgical failure in both the nasal mucosa and lacrimal sac. All 15 patients without fibrosis in the nasal mucosa had surgical success $(100 \%)$, and 14 of 15 patients without fibrosis in lacrimal sac showed a surgical success (93.3\%). HSP47 expression in the nasal mucosa was found in 21 of 30 patients, and no severe expression was found. HSP47 expression in lacrimal sac tissue was found in 15 of 30 patients, and severe expression was found in 2 patients. Higher HSP47 expression in both the nasal mucosa and lacrimal sac tissue was correlated with surgical failure.

HSP47 expression causes collagen accumulation, which causes tissue fibrosis. In nasal mucosa and lacrimal sac tissue, higher HSP47 expression was correlated with higher degrees of fibrosis. However, we found no correlation between HSP47 expression and the occurrence and size of the granuloma formed in the ostium region after surgery.

The present study has limitations, as it was conducted with a relatively small sample size, and an approximate analysis method, the linear by linear association method, was used to analyze the trend. However, by histologically analyzing the nasal mucosa and lacrimal sac for the first time, we found that fibrosis and squamous metaplasia affected the outcome of DCR surgery. In addition, HSP47 expression is highly correlated with fibrosis in the nasal mucosa and lacrimal sac and may directly and indirectly affect the outcome of DCR surgery.

\section{Summary}

\section{What was known before}

- The heat shock protein 47 (HSP47) is closely associated with the fibrosis of a tissue.

- HSP47 can be expressed in the nasal mucosa of patients who had undergone endoscopic surgery.

\section{What this study adds}

- Fibrosis and squamous metaplasia of the nasal mucosa and lacrimal sac lower the success rate of endoscopic dacryocystorhinostomy (EDCR).

- Fibrosis is correlated with the expression of HSP47.
- The expression of HSP47 in nasal mucosa and lacrimal sac lowers the success rate of EDCR.

Funding This study was supported by a Korea University Grant. This study was approved by the Institutional Review Board of Korea University Guro Hospital.

Author contributions Conception and design of the study (JP, SB); conduction of study (JP, JL, SB); collection and management of data (JP, JL, SB); data analysis (JP, JL, SB); data interpretation (JP, JL, $\mathrm{SB}$ ); and preparation, review, and approval of the manuscript (JP, JL, $\mathrm{SB})$.

\section{Compliance with ethical standards}

Conflict of interest The authors declare that they have no conflict of interest.

\section{References}

1. Tarbet KJ, Custer PL. External dacryocystorhinostomy. Surgical success, patient satisfaction, and economic cost. Ophthalmology. 1995;102:1065-70.

2. McDonogh M, Meiring JH. Endoscopic transnasal dacryocystorhinostomy. J Laryngol Otol. 1989;103:585-7.

3. Durvasula VS, Gatland DJ. Endoscopic dacrocystorhinostomy: long-term results and evolution of surgical technique. J Laryngol Otol. 2004;118:628-32.

4. Fayet B, Racy E, Assouline M. Complications of standardized endonasal dacryocystorhinostomy with unciformectomy. Ophthalmology. 2004;111:837-45.

5. Gupta A, Prabhakaran VC, Dodd T, Selva D. Characterization of lacrimal sac histology: an immunohistochemical study. Clin Exp Ophthalmol. 2012;40:869-73.

6. Xiang XH, Jiang TP, Zhang S, Song J, Li X, Yang JY, et al. Pirfenidone inhibits proliferation, arrests the cell cycle, and downregulates heat shock protein- 47 and collagen type I in rat hepatic stellate cells in vitro. Mol Med Rep. 2015;12: 309-14.

7. Smirnov G, Pirinen R, Tuomilehto H, Seppa J, Terasvirta M, Uusitalo H, et al. Strong expression of HSP47 in metaplastic nasal mucosa may predict a poor outcome after primary endoscopic dacryocystorhinostomy: a prospective study. Acta Ophthalmol. 2011;89:132-6.

8. Mauriello JA Jr., Palydowycz S, DeLuca J. Clinicopathologic study of lacrimal sac and nasal mucosa in 44 patients with complete acquired nasolacrimal duct obstruction. Ophthal Plast Reconstr Surg. 1992;8:13-21.

9. Jang SY, Lee KH, Lee SY, Yoon JS. Effects of nasopore packing on dacryocystorhinostomy. Korean J Ophthalmol. 2013;27: 73-80.

10. Ozkiriş M, Ozkiriş A, Göktaş S. Effect of mitomycin C on revision endoscopic dacryocystorhinostomy. J Craniofac Surg. 2012;23:608-10.

11. Berlucchi M, Castelnuovo P, Vincenzi A, Morra B, Pasquini E. Endoscopic outcomes of resorbable nasal packing after functional endoscopic sinus surgery: a multicenter prospective randomized controlled study. Eur Arch Otorhinolaryngol. 2009;266:839-45.

12. Ciftci F, Erşanli D, Civelek L, Baloqlu H, Karadayi K, Gungor A. Histopathologic changes in the lacrimal sac of dacryocystorhinostomy patients with and without silicone intubation. Ophthal Plast Reconstr Surg. 2005;21:59-64. 
13. Chen JJ, Jin PS, Zhao S, Cen Y, Liu Y, Xu XW, et al. Effect of heat shock protein 47 on collagen synthesis of keloid in vivo. ANZ J Surg. 2011;81:425-30.

14. Taguchi T, Razzaque MS. The collagen-specific molecular chaperone HSP47: is there a role in fibrosis? Trends Mol Med. 2007;13:45-53.

15. Razzaque MS, Taguchi T. The possible role of colligin/HSP47, a collagen-binding protein, in the pathogenesis of human and experimental fibrotic diseases. Histol Histopathol. 1999;14: 1199-212.

16. Razzaque MS, Taguchi T. Role of glomerular epithelial cellderived heat shock protein 47 in experimental lipid nephropathy. Kidney Int Suppl. 1999;71:256-9.

17. Xia Z, Abe K, Furusu A, et al. Suppression of renal tubulointerstitial fibrosis by small interfering RNA targeting heat shock protein 47. Am J Nephrol. 2008;28:34-46. 